\title{
Fellow Column: \\ Diagnostic Precision in Neonatal Medicine: Why Over Investigate?
}

Adil Khan, MD, Shabih Manzar, MD

\section{Abbreviations:}

CMV (cytomegalovirus), TORCH (Toxoplasmosis, others, rubella, C, Herpes), IUGR (intra

"Due to an increased reliance on the investigation, the art of clinical medicine is disrupted. The disruption in skill acquisition and critical thinking is concerning. A medical student's journey from a clerkship to practice should be based on acquiring clinical skills and applying critical thinking. In the field of neonatology, it has been observed that the investigation is a rule rather than an exception."

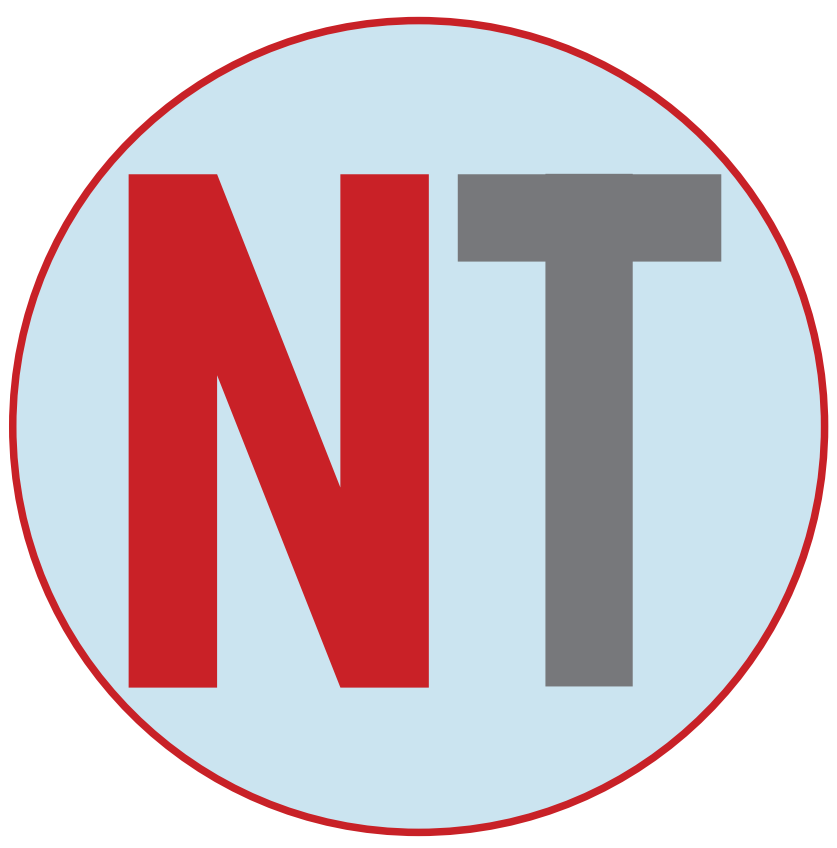

uterine growth restriction).

\section{Introduction:}

Neonatal patients are unique. They cannot express symptoms; however, a vigilant clinical examination may elicit signs that could be helpful in diagnosis. Due to an increased reliance on the investigation, the art of clinical medicine is disrupted. The disruption in skill acquisition and critical thinking is concerning. A medical student's journey from a clerkship to practice should be based on acquiring clinical skills and applying critical thinking. In the field of neonatology, it has been observed that the investigation is a rule rather than an exception. Here we present an example of a neonate who was over investigated for thrombocytopenia.

\section{Case study:}

A newborn male infant was admitted to the nursery with an initial platelet count of $73,000 \mathrm{~K} / \mathrm{uL}$. He was delivered via cesarean section at 38 weeks of gestation to a 34-year-old mother, gravida 4 , Para 3103 . The pregnancy was complicated by pregnancyinduced immune thrombocytopenia (ITP) for which she received a 10-day course of oral prednisolone. Her prenatal labs revealed a negative sickle cell screen, HIV, hepatitis B, and syphilis. She was rubella immune. Her blood group was $O$ positive with negative antibodies. She had a past medical history of herpes simplex virus, treated with acyclovir. Upon delivery, the baby had a strong cry, he was dried, stimulated, and bulb suctioned. Apgar score was 8 and 9 at one and five minutes, respectively. His birth weight was 2375 grams (3.5\%) and head circumference of $32.5 \mathrm{~cm}(16 \%)$. No signs of bleeding or bruises were noted. During rounds, a decision was made based on the clinical pathway to obtain an extensive workup to look for the reasons for low platelets. Although, this was a classic case of autoimmune thrombocytopenia, a TORCH titer, 3-daily urine CMV tests, and ultrasound of the head and abdomen were ordered. The natural resolution and course are depicted in Figure 1. What was the message given to the trainee?

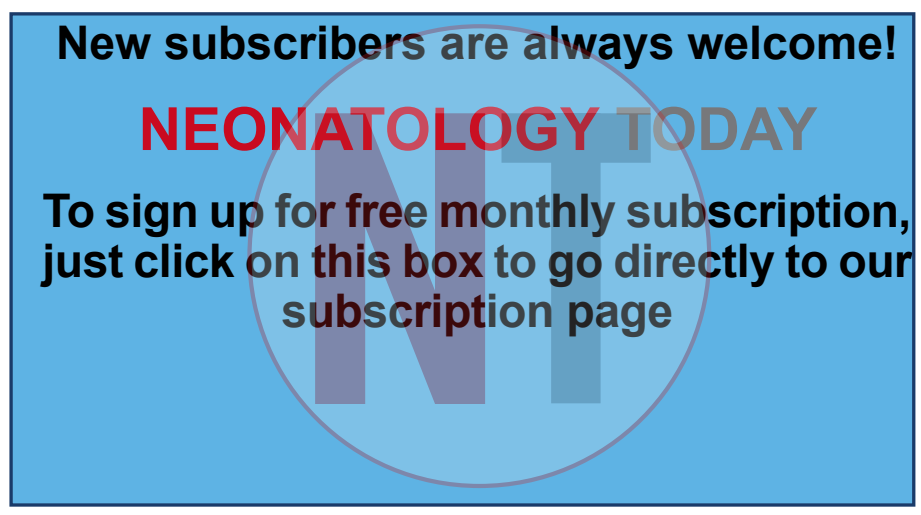




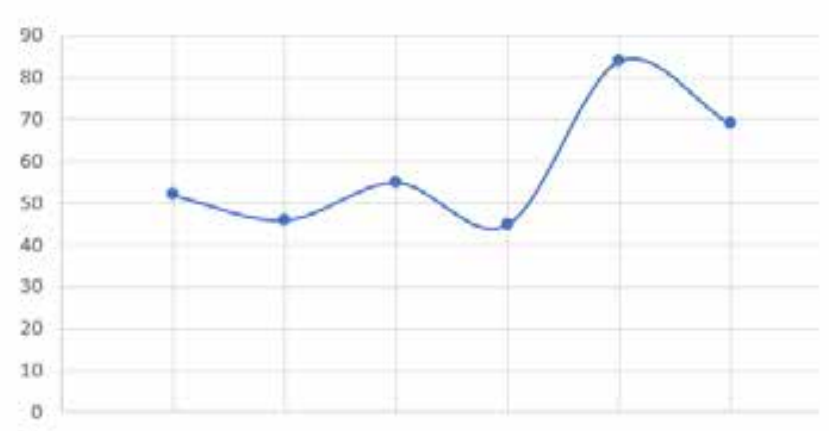

Maternal Platelet Count (K/uL)

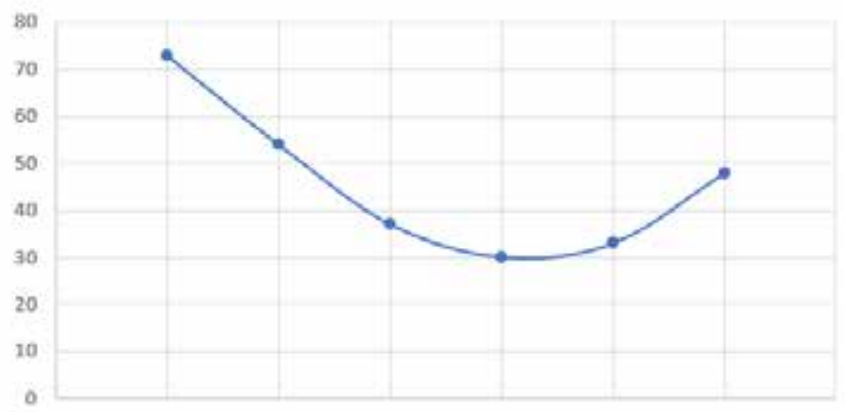

Infant Platelet Count (K/uL)
Figure1: Maternal and Infant platelet count (K/uL) over time

\section{Discussion:}

Instead of following an approach of good history taking, developing a list of differential diagnoses, narrowing it out to the most likely diagnosis, and then investigating, the infant had multiple tests. In hospital practice, to minimize variation among practitioners' clinical pathways are often used. This approach has shown to contain costs and decrease the length of stay while maintaining clinical outcomes. (1) The downside of developing these algorithms is taking away the critical thinking process among medical students and young practitioners.
The best clinical decision refers to making a choice that maximizes effectiveness and minimizes harm. (2) A practitioner who is doubtful will end up maximizing the investigations. If a provider's decision-making has built-in subjectively and lack of evidence and skills, this may result in variation and unpredictability. (3) To minimize these problems, using a decision tree could be a potential solution. (4)

As noted in the case, there was no indication for obtaining TORCH titers and urine CMV PCR. The infant was borderline small for gestational age (BW $3.5 \%$ ) with a head circumference of $16 \%$. Maternal serology was negative for syphilis, and she was rubella immune. An infant with TORCH infection presents with symmetrical IUGR. CMV could be asymptomatic at birth, but in this case,

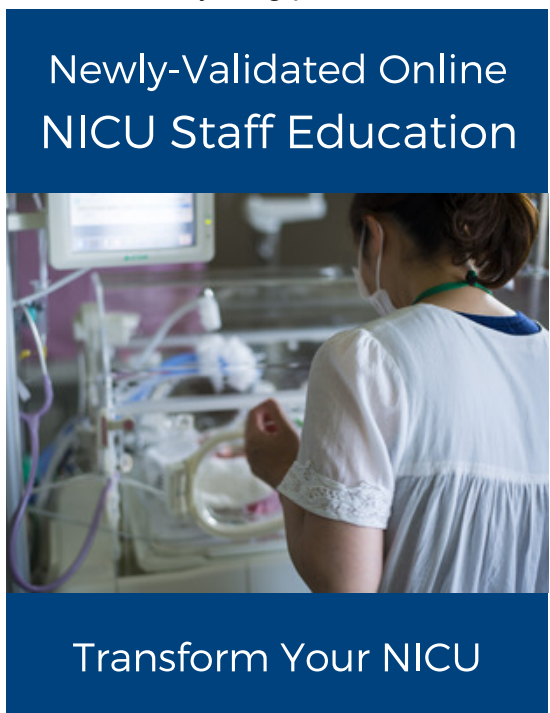

Caring for Babies and their Families: Providing Psychosocial Support to NICU Parents

\section{based on the "Interdisciplinary Recommendations for Psychosocial} Support for NICU Parents."

\section{Contact sara@mynicunetwork.com for more information.}

\section{Brought to you by a collaboration between}

- National Perinatal Association

- Patient + Family Care

- Preemie Parent Alliance

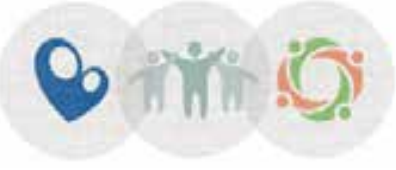

www.mynicunetwork.com 
there was compelling evidence that TP was immune-mediated.

Now let us look at the multiple-choice question (MCQ) as an example (Appendix). All answers are correct, but with the knowledge and critical thinking, one can delineate to the best response. Similarly, the investigation should be tailored to the most likely diagnosis. A good history and clinical examination should eliminate the need for extensive investigation.

To conclude, as clinicians and medical educators, we should practice and teach our students the art of good history taking and to perform a complete physical examination. They should apply knowledge and critical thinking rather than jumping on extensive investigations. We should try to minimize the disruption in medical education and clinical practice.

\section{References:}

1. Lion KC, Wright DR, Spencer S, Zhou C, Del Beccaro M, Mangione-Smith R. Standardized clinical pathways for hospitalized children and outcomes. Pediatrics. 2016;137(4). pii: e20151202. doi:10.1542/peds.2015-1202.

2. Aleem IS, Schemitsch EH, Hanson BP. What is a clinical decision analysis study? Indian J Orthop. 2008; 42:137-9.

3. Banning M.A review of clinical decision making: models and current research. J Clin Nurs. 2008; 17:187-95.

4. Bae JM. The clinical decision analysis using decision tree. Epidemiol Health. 2014;36: e2014025.

\section{Appendix}

A term newborn is noted to have a platelet count of $74 \mathrm{~K} / \mathrm{uL}$. Mother has a history of immune thrombocytopenia. The best next management step is:
A) Obtain TORCH titer and urine CMV
B) Obtain father's platelet antigen genotype
C) Follow serial head ultrasound
D) Follow serial platelet count
E) Transfuse platelets for count $<50 \mathrm{~K} / \mathrm{uL}$

Correct Answer: D

In Practice: $A, B, C, D, E$ (just in case, what if)

Early Neonatal thrombocytopenia ( $<72$ hours of life) could be due to intrauterine infection or immune-mediated. When the mother has ITP, the antibodies cross the placenta and destroy fetal platelet resulting in thrombocytopenia (TP). An infant born with a suspected infection should be checked for TORCH titer and urine CMV. However, isolated TP in the neonate is most likely immunemediated. When maternal platelets are normal, alloimmune TP (NAIT) should be considered high in the list of differential diagnoses. When it comes to treatment, platelet transfusion is reserved for platelet count $<25 \mathrm{~K} / \mathrm{uL}$ in well infants. Following serial head ultrasound is not logical. A screening HUS may be done, especially in NAIT. Decision pathways and management algorithms should be used as a guide; however, the investigation should not include all.

1. Del Vecchio A. Evaluation and management of thrombocytopenic neonates in the intensive care unit. Early Hum Dev. 2014;90 Suppl 2: S51-S55.
2. Curley A, Stanworth SJ, Willoughby K, et al. A Randomized Trial of Neonatal Platelet Transfusion Thresholds in Neonates. N Engl J Med. 2019;380: 242-251.

Funding Source: None

Financial Disclosure: None

Conflict of Interest: None

Contributor Statement:

Dr. Adil Khan is a second-year pediatrics resident. He has written the report and Dr. Shabih

Manzar has provided mentorship and has reviewed the manuscript.

NT

Fellow's Column is published monthly.

- Submission guidelines for "Fellow's Column":

- 2000 word limit not including references or title page. Exceptions will be made on a case by case basis

- $\quad$ QI/QA work, case studies, or a poster from a scientific meeting may be submitted.

- Submission should be from a resident, fellow, or NNP in training.

- Topics may include Perinatology, Neonatology, and Younger Pediatric patients.

- No more than 20 references.

- $\quad$ Please send your submissions to:

Elba Fayard, MD

Interim Fellowship Column Editor LomaLindaPublishingCompany@gmail.com

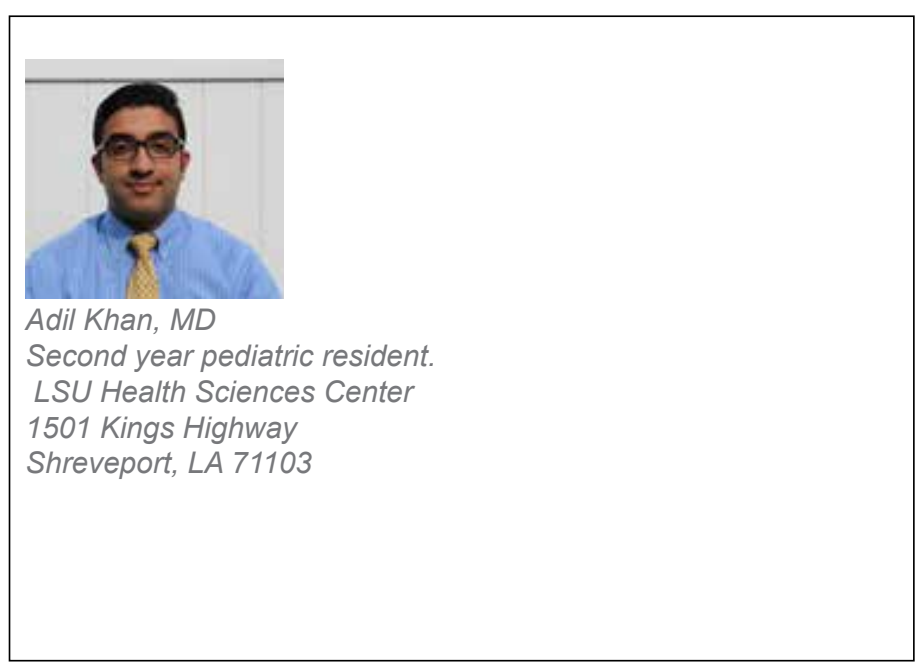



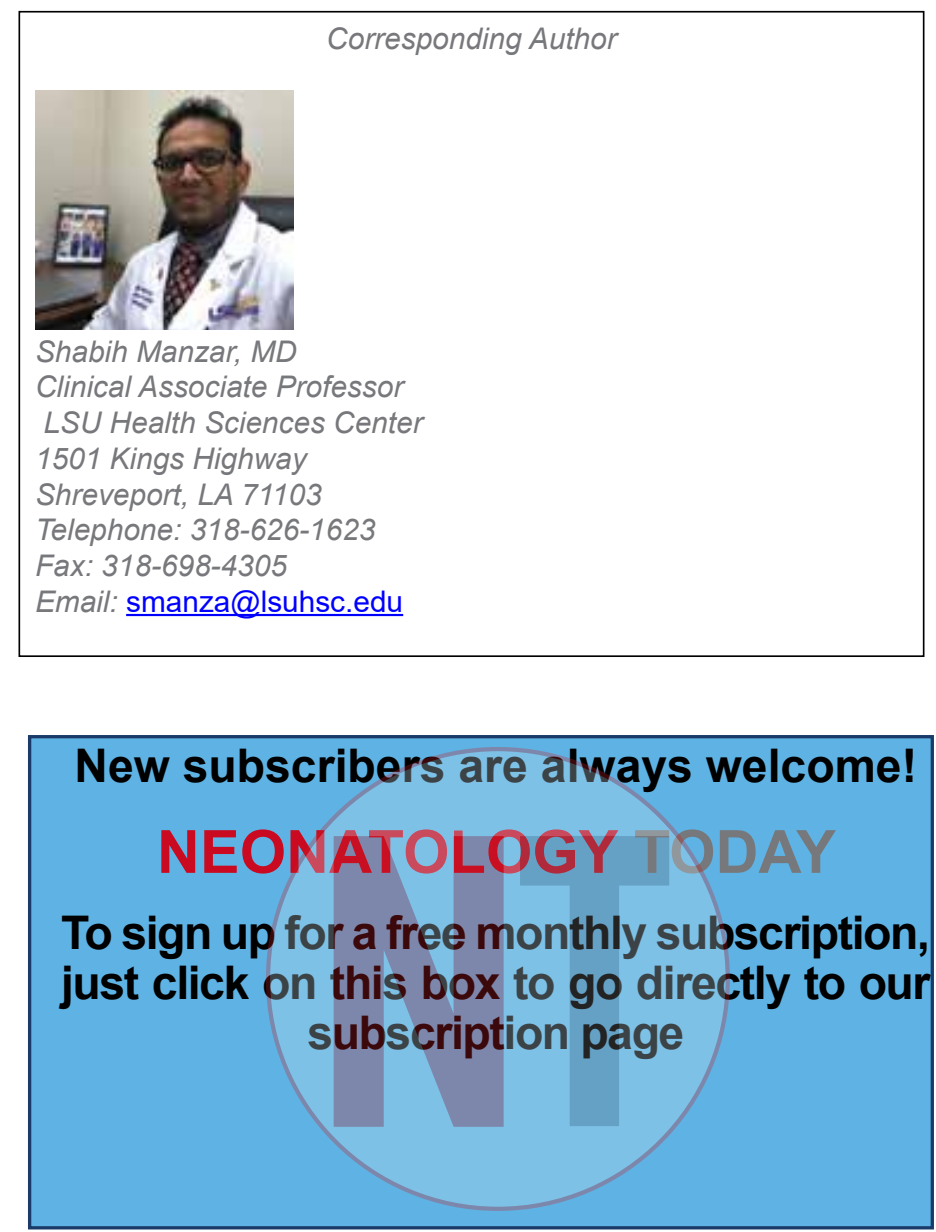

\section{Readers can also follow NEONATOLOGY \\ via our Twitter Feed @NEOTO}

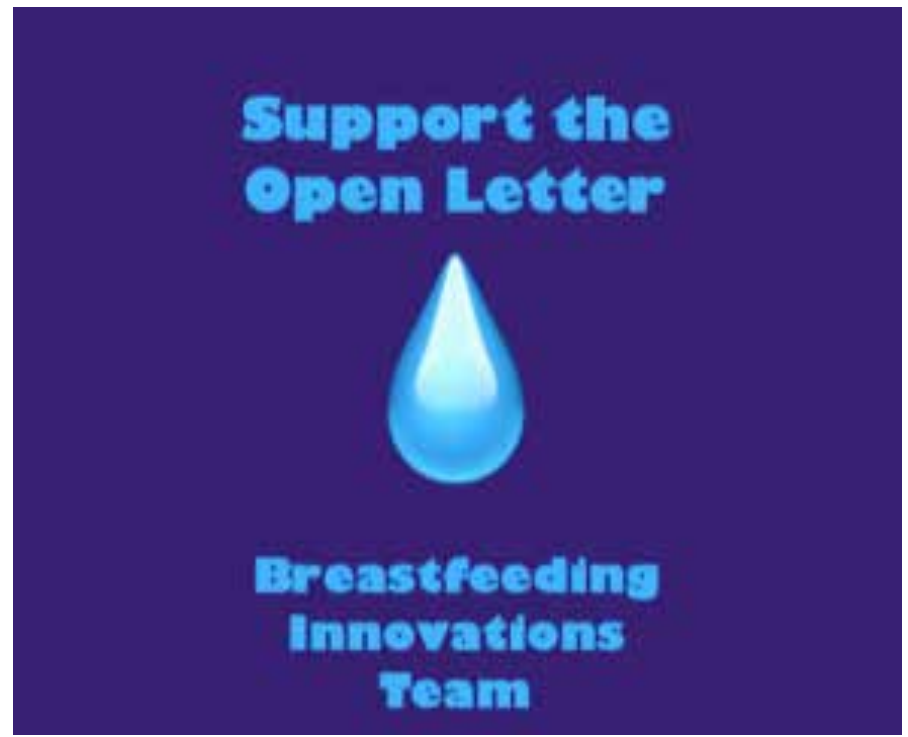

SAFETY IN THE NICU New tubes, new problems?

A new tubing design meant to eliminate tubing misconnections has introduced new challenges for the NICU population. Pediatric providers must deliver medication in small volumes to tiny patients with high levels of accuracy. The new tubing design, known as ENFit ${ }^{\oplus}$, could present dosing accuracy and workflow challenges.
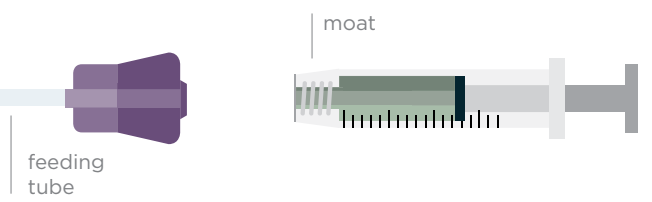

\section{DOSING ACCURACY}

- The moat, or area around the syringe barrel, is difficult to clear. Medication can hide there, inadvertently increasing the delivered dose when the syringe and feeding tube are connected; patients may receive extra medication.

\section{INFECTION RISK}

- The moat design can increase risk for infection if residual breast milk or formula remains in the moat and transfers to the feeding tube.

\section{WORKFLOW ISSUES}

- Increased nursing workflow is seen with additional steps for clearing syringe moats, cleaning tube hubs, and using multiple connectors.

Improved standards are important to protect patients from the dangers of tubing misconnections. But we must avoid mitigating existing risks by creating new ones.

Individual hospitals should consider all factors impacting their NICU patients before adopting a new tubing design.

ENFit ${ }^{\oplus}$ is a registered trademark of GEDSA

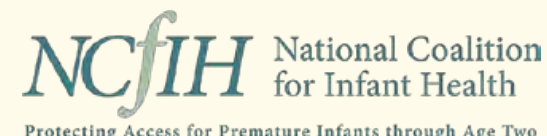

A collaborative of professional, clinical, community health, and family support organizations focused on the health and safety of premature infants.

\section{infanthealth.org}

